\title{
Modifiable factors within the prevention of mother-to-child transmission programme associated with failure to prevent HIV transmission in the Onandjokwe district of Namibia
}

\author{
Flavia Strato Shayo and Bob Mash* (D) \\ Division of Family Medicine and Primary Care, Stellenboch University, Stellenbosch, South Africa \\ *Corresponding author, email: rm@sun.ac.za
}

Background: Ending new paediatric HIV infections continues to be a global health priority. Cuba and other countries have demonstrated that elimination of mother-to-child transmission is possible through Prevention of Mother-to-Child Transmission (PMTCT) interventions. As Namibia works on improving PMTCT there is a need to identify the local modifiable factors to achieve zero new HIV infections.

Aim: This study aimed to identify the modifiable factors within the PMTCT programme, which contributed to the acquisition of HIV infection among children.

Setting: The study was carried out in the Onandjokwe District, Northern Namibia.

Methods: A descriptive audit was undertaken of 59 medical records of mothers and their children under two years, who acquired HIV despite the PMTCT programme between 2014 and 2016.

Results: The study found that overall HIV transmission was only $2 \%$, but $80 \%$ of the paediatric HIV infections could be prevented by implementing the existing Namibian PMTCT recommendations. Overall $61 \%$ of modifiable factors were related to mothers, $30 \%$ to health workers and $10 \%$ to the health system. The top three modifiable factors were the mother defaulting on ART during pregnancy or breastfeeding, the health worker not intervening when the mother failed the first-line ART regimen, and poor coordination of care between the hospital and primary care.

Conclusion: Although overall transmission is low with the PMTCT programme, the majority of remaining HIV infections among children under two years could be prevented by addressing the modifiable factors identified in this study.

Keywords: antenatal care, communicable disease transmission, human immunodeficiency virus, infant, Namibia, pregnancy, risk factors

\section{Introduction}

The elimination of HIV by preventing mother-to-child transmission continues to be a global health priority. ${ }^{1}$ The third United Nations Sustainable Development Goal (SDG) includes a commitment to end the AIDS epidemic. ${ }^{2}$ One of the major steps in getting to zero new HIV infections is ending paediatric HIV infections. Mother-to-child transmission has been virtually eliminated in high-income countries and demonstrates that the use of appropriate antiretroviral (ARV) medication during pregnancy and breastfeeding can reduce the risk of vertical transmission to less than $5 \%{ }^{3}$ Cuba, a low-income country, has demonstrated that it is possible to eliminate HIV infection in children through the Prevention of Mother-To-Child Transmission (PMTCT) programme, as it became the first country certified by the World Health Organization (WHO) in 2015 to have done so. ${ }^{4}$ To date four more countries (Thailand, Belarus, Armenia and Moldova) have been validated by the WHO. ${ }^{5}$ Eliminating new HIV infections among children through the PMTCT programme has been shown to be cost-effective as it decreases morbidity and mortality, the psychological burden of HIV, as well as economic costs to both individuals and society. ${ }^{6}$

The PMTCT programme has been proven to effectively reduce the risk of transmitting HIV infection from mothers to their babies, even in low-resource settings. ${ }^{7,8}$ The benefit of using a short-term combination of triple ARVs in pregnant mothers and single-dose nevirapine in exposed infants was shown to significantly decrease the chance of a mother transmitting HIV to her baby. ${ }^{9}$ WHO guidelines for PMTCT in 2013 and 2015 recommended starting life-long Highly Active Anti-Retroviral Therapy (HAART) in all HIV-positive pregnant and breastfeeding mothers and to offer nevirapine to their babies throughout the breastfeeding period. ${ }^{9,10}$ Adoption of the new 2016 WHO guideline recommendations to treat everyone who tests HIV-positive is expected to further reduce the chance that children born to HIV-positive mothers will be HIV infected because of earlier initiation of ARV and attainment of viral suppression. ${ }^{10,11}$ Prevention of new infections through the PMTCT programme will not only save the life of the baby, but will keep the mother alive by giving her the treatment she needs, and protect other children in the family from being orphaned. Ongoing efforts to stop new HIV infections emphasise the need for frequent monitoring of HIV viral loads among pregnant and breastfeeding mothers, use of dual prophylaxis (AZT and nevirapine) for infants at higher risk of HIV acquisition and repeated regular HIV testing of HIV-negative mothers during the breastfeeding period to close the remaining gaps in PMTCT. ${ }^{12}$

Namibia has implemented PMTCT since 2005 and the latest Namibian guidelines adopt the 2016 WHO recommendations, with a clear commitment to the elimination of mother-to-child transmission. ${ }^{13}$ Identifying avoidable factors related to HIV transmission during the PMTCT period can help the health services to achieve this goal. Some of the known modifiable risk factors in the PMTCT programme in sub-Saharan countries are poor access to maternal care and/or PMTCT services due to 
geographic factors, non-availability of medication, economic barriers such as transport costs, and unacceptable services due to negative staff attitudes. ${ }^{14,15}$ The quality of care may also be impaired due to a failure to offer PMTCT and/or a lack of knowledge amongst healthcare professionals. ${ }^{16}$ The modifiable factors may differ between countries and even districts within countries and therefore local modifiable factors must be identified and solutions developed to prevent new HIV infections in children. ${ }^{17-19}$ This type of research has yet to be conducted in Namibia.

A study at Onandjokwe Hospital, Namibia in 2013 showed that $13 \%$ of mothers of children less than five years old, who were admitted and died, were not given an HIV test during pregnancy and $15 \%$ of the children that died were confirmed HIV-positive for the first time when admitted to the ward. ${ }^{20,21}$ From February 2014 until January 2016 a total of 67 children under two years of age acquired HIV despite the PMTCT programme at Onandjokwe Hospital. This study in the Onandjokwe District aimed to identify the modifiable factors within the PMTCT programme, which contributed to the acquisition of HIV infection among these children. The identified modifiable factors can be used by PMTCT stakeholders to design local strategies to close the gaps and prevent new paediatric HIV infection.

\section{Methods}

\section{Study design}

The study was a descriptive audit of the medical records of mothers and their children under two years, who acquired HIV despite the PMTCT programme's existence in the period 20142016.

\section{Study setting}

The study was conducted at Onandjokwe District in northern Namibia, located $750 \mathrm{~km}$ from the capital city of Windhoek. Onandjokwe District covers approximately $25000 \mathrm{~km}^{2}$ with a catchment population of 147000 . The majority of patients live in rural villages and walk to nearby health facilities. The district has one referral hospital (Onandjokwe), three health centres and eight primary care clinics.

All facilities were implementing PMTCT option B+ since 2014 as part of antenatal care (ANC) services. The PMTCT option B+ offers all pregnant and breastfeeding women HAART as soon as they are diagnosed, which is continued for life regardless of CD4 count. Prior to 2014 Option A was given to pregnant mothers who did not quality for ART. ${ }^{22}$

Onandjokwe District Hospital offers the PMTCT services via antenatal and intrapartum care and has ARVs easily available. Onandjokwe's ART clinic is the third largest in the country by patient volume and serves as the referral centre for the rest of the 11 healthcare facilities in the district that offer HIV services. Deliveries are all conducted at the hospital and the primary care facilities also provide antenatal, postnatal and HIV care. HIV testing is performed by health assistants, who may not always be available at the clinics or at night when emergencies are seen.

Exposed infants received nevirapine at their respective clinics and were followed up until the age of six weeks when their first DNA polymerase chain reaction (PCR) test was performed; those who tested HIV-positive were referred to Onandjokwe for confirmation and prepared for ART initiation. All children who tested DNA PCR negative at 6 weeks continued with breastfeeding up to 12 months while receiving nevirapine. A second diagnostic rapid test (Determine) was performed at nine months and repeated four weeks after stopping breast milk exposure. Any child who tested positive by HIV rapid test was referred to Onandjokwe Hospital for an HIV confirmatory test and was then started on ART. All paediatric patients were initiated on ART at Onandjokwe Hospital and monitored for at least six months or until the first viral load result was reviewed. Those with suppressed viral load and who were clinically stable were then referred, with their mothers, to a nearby health facility for continued care.

\section{Study population}

The population included all children who were confirmed HIVpositive in the chronic care ART register, were under the age of two years, and registered between April 1, 2014 and March 30, 2016 at Onandjokwe District Hospital. The register showed a total of 67 children for this period.

\section{Data collection}

A standardised data collection tool was used to collect data from each individual record on adherence to the key recommendations of the PMTCT guidelines during pregnancy, delivery and postpartum care.

The adherence to ART was assessed by healthcare workers using pill counts, whereby patients who consumed more than $95 \%$ of the recommended doses supplied at the last visit were regarded as having good adherence, between $85 \%$ and $95 \%$ as having fair adherence and those at less than $85 \%$ were classified as having poor adherence. Any patient who missed his/her clinic visit and interrupted ART treatment was defined as a 'defaulter' and should have received counselling to avoid further interruptions. Any patient who interrupted ART for 90 consecutive days or more was defined as 'lost to follow-up' and should have been traced, brought to care and reasons explored to prevent future problems. ${ }^{13}$

The design of the tool was based on another study at Onandjokwe Hospital on the causes of mortality and associated modifiable healthcare factors for children $\left(<5\right.$ years) ${ }^{20}$ The tool was submitted to a panel of experts to validate its content and construct and was piloted on six test cases to ensure it was practical to collect data. The tool consisted of the following information: demographic information, PMTCT option, infant feeding choice, date when mother initiated ARV therapy, overall adherence to ARVs of the mother, date when the child was confirmed HIV-positive and the HIV viral load of the mother.

Data were extracted by the principal investigator. The medical records reviewed included the ANC registers, labour and delivery register books, maternity delivery clinical notes, postnatal clinical notes, mother-baby care follow-up register, and ART patient care booklets.

Data captured in the tool were then analysed by a review team who reached a consensus on any reason(s) why the PMTCT programme failed to prevent transmission and a judgement as to whether the infant's HIV infection could have been prevented. The review team included the principal investigator, senior HIV doctor and the district HIV clinical mentor. This team also referred to the records themselves if necessary to clarify issues. 


\section{Data analysis}

Data were captured on an Excel spreadsheet (Microsoft Corp, Redmond, WA, USA) and checked for any missing, incomplete or inconsistent data before analysis. The data were analysed by a statistician using the Statistical Package for the Social Sciences version 16.0 (SPSS Inc, Chicago, IL, USA). Descriptive statistics were used to determine frequencies and percentages or means and standard deviations.

\section{Results}

The study included 59 children out of the possible 67 , due to 8 missing records. A total of 19898 deliveries were conducted during the study period, among whom 3358 pregnant mothers were HIV-positive $(17 \%)$ and the transmission rate was therefore $2 \%$.

Of the 59 children the majority were female with a normal vaginal delivery (Table 1 ). Although $42 \%$ of mothers started on ART before pregnancy and a further 19\% during pregnancy, $39 \%$ of these mothers started ART only at delivery or in the postnatal period.

Table 2 presents the PMTCT options used and infant feeding practices. As expected the majority of mothers received PMTCT Option B + . One mother was still on Option A and following the previous guidelines. The majority of infants (82\%) did not receive nevirapine as they should, 51\% never received

Table 1: Characteristics of study children and their mothers $(n=59)$

\begin{tabular}{|c|c|c|}
\hline Characteristics & $n$ & $\%$ \\
\hline \multicolumn{3}{|l|}{ Child gender: } \\
\hline Male & 21 & 35.6 \\
\hline Female & 38 & 64.4 \\
\hline \multicolumn{3}{|l|}{ Mode of delivery: } \\
\hline Normal vaginal delivery & 54 & 91.5 \\
\hline Caesarean section & 5 & 8.5 \\
\hline \multicolumn{3}{|l|}{ Place of delivery: } \\
\hline Health facility & 48 & 81.4 \\
\hline Home & 8 & 13.6 \\
\hline In transit & 3 & 5.1 \\
\hline \multicolumn{3}{|l|}{ Level of education of mothers: } \\
\hline Primary & 18 & 0.5 \\
\hline Secondary & 38 & 64.4 \\
\hline College & 1 & 1.7 \\
\hline None & 2 & 3.4 \\
\hline \multicolumn{3}{|l|}{ Time of HIV diagnosis of mothers: } \\
\hline Before pregnancy & 29 & 49.2 \\
\hline During first trimester & 3 & 5.1 \\
\hline During second trimester & 5 & 8.5 \\
\hline During third trimester & 5 & 8.5 \\
\hline During labour and delivery & 2 & 3.4 \\
\hline During postnatal and breastfeeding & 15 & 25.4 \\
\hline \multicolumn{3}{|l|}{ Time of ART initiation for mothers: } \\
\hline Before pregnancy & 25 & 42.4 \\
\hline 1st trimester & 3 & 5.1 \\
\hline 2nd trimester & 5 & 8.5 \\
\hline 3rd trimester & 3 & 5.1 \\
\hline Labour and delivery & 1 & 1.7 \\
\hline Postnatal and breastfeeding period & 22 & 37.3 \\
\hline
\end{tabular}

Table 2: PMTCT and feeding options $(n=59)$

\begin{tabular}{lrr}
\hline Variables & $\boldsymbol{n}$ & $\%$ \\
\hline Mother's PMTCT options: & 1 & 1.7 \\
\hline Allocated to PMTCT Option A & 43 & 72.9 \\
\hline Allocated to PMTCT Option B+ & 15 & 25.4 \\
\hline No PMTCT allocation during pregnancy & & \\
\hline Children PMTCT options: & 18 & 30.8 \\
\hline Children received nevirapine during delivery only & 11 & 18.6 \\
\hline Children received nevirapine during delivery and & & \\
breastfeeding & 30 & 50.8 \\
\hline Children never received nevirapine & & \\
\hline Mother's choice of feeding option after delivery: & 57 & 96.6 \\
\hline Breastfeeding & 2 & 3.4 \\
\hline Formula milk feeding & \multicolumn{3}{|c|}{} \\
\hline Infant feeding practices: & 32 & 54.2 \\
\hline Exclusive breastfeeding until 6 months & 26 & 44.1 \\
\hline Mixed feeding & 1 & 1.7 \\
\hline Formula milk &
\end{tabular}

nevirapine and a further $31 \%$ only received it at delivery. Although $97 \%$ of mothers intended to breastfeed exclusively only $54 \%$ actually did and $44 \%$ mixed fed their infants under the age of 6 months.

Table 3 presents the key characteristics of mothers' ART at the point when their child was diagnosed with HIV. Among this group of mothers $51 \%$ had been on ART for more than 6 months and of those $63 \%$ were poorly adherent to treatment and $67 \%$ never had their viral load checked, mainly because they did not attend the follow-up appointments.

Overall, 279 modifiable factors were identified and the majority of these factors $(61 \%)$ were related to the mother (Table 4). Most of these factors were associated with poor adherence to ART in the mother or their infant (28\%), poor adherence with antenatal care visits $(13 \%)$, mixed feeding (9\%) and unsafe sex (8\%). In addition, $30 \%$ of the factors were related to the clinical care provided by health professionals. Key issues in the health professionals included a failure to check for or respond to poor adherence to ART (19\%), a delay in initiating ART in the

Table 3: Mothers' follow-up care characteristics

\begin{tabular}{lcc}
\hline Variables & $n$ & $\%$ \\
\hline Mother on ART follow-up $\geq 6$ months $(n=59):$ & 30 & 50.8 \\
\hline Yes & 29 & 49.2 \\
\hline No & 5 & \\
\hline Adherence in those on ART follow-up $\geq 6$ months $(n=30):$ & 16.7 \\
\hline Good & 6 & 20.0 \\
\hline Fair & 19 & 63.3 \\
\hline Poor & 2 & \\
\hline Viral load at 6 months post ART initiation $(n=30):$ & 6.7 \\
\hline$<40$ IU/ml & 1 & 3.3 \\
\hline $40-1$ 000 IU/ml & 7 & 23.3 \\
\hline 1 000 IU/ml & 20 & 66.7 \\
\hline Viral load not done & & \\
\hline Reasons for the viral load not done $(n=20):$ & 20.0 \\
\hline Unknown & 4 & 80.0 \\
\hline Missed follow-ups & 16 & \\
\hline
\end{tabular}


Table 4: Modifiable factors in mother-to-child transmission $(n=279)$

\begin{tabular}{|c|c|c|}
\hline Modifiable factors & $n$ & $\%$ \\
\hline Modifiable factors related to healthcare system: & 27 & 9.7 \\
\hline $\begin{array}{l}\text { No system for clinics to identify and follow up postnatal } \\
\text { mothers }\end{array}$ & 25 & 9.0 \\
\hline Nevirapine out of stock & 2 & 0.7 \\
\hline Modifiable factors related to healthcare professionals: & 83 & 29.7 \\
\hline $\begin{array}{l}\text { Patient was failing first-line ART regimen with no } \\
\text { intervention }\end{array}$ & 36 & 12.9 \\
\hline Failed to check viral load & 4 & 1.4 \\
\hline Failed to trace the patients who defaulted on ART & 14 & 5.0 \\
\hline Mother attended ANC, but not offered HIV test & 10 & 3.6 \\
\hline $\begin{array}{l}\text { Pregnant mother tested HIV-positive, but ART initiated } \\
\text { later in pregnancy or only when a child tested positive }\end{array}$ & 10 & 3.6 \\
\hline $\begin{array}{l}\text { The new born was not given NVP until follow up at } 6 \\
\text { weeks }\end{array}$ & 8 & 2.9 \\
\hline $\begin{array}{l}\text { Healthcare professionals offered HIV test very late in } \\
\text { pregnancy }\end{array}$ & 1 & 0.4 \\
\hline Modifiable factors related to mother and children: & 169 & 60.6 \\
\hline $\begin{array}{l}\text { Mother defaulted on ART during pregnancy and } \\
\text { breastfeeding }\end{array}$ & 34 & 12.2 \\
\hline Mixed feeding started before infant reached 6 months & 26 & 9.3 \\
\hline Mother had high viral load before pregnancy & 22 & 7.9 \\
\hline Mother defaulted on nevirapine treatment in the infant & 21 & 7.5 \\
\hline Mother did not attend appointment for viral load check & 16 & 5.7 \\
\hline $\begin{array}{l}\text { Mother seroconverted during pregnancy or } \\
\text { breastfeeding }\end{array}$ & 15 & 5.4 \\
\hline Mother never attended ANC & 11 & 3.9 \\
\hline Mother started ANC very late, HIV test done very late & 8 & 2.9 \\
\hline Mother became pregnant while preparing to start ART & 8 & 2.9 \\
\hline Delivered at home & 7 & 2.5 \\
\hline $\begin{array}{l}\text { Mother tested positive, but refused to be initiated on } \\
\text { ART }\end{array}$ & 1 & 0.4 \\
\hline
\end{tabular}

mother or prophylaxis in the infant (7\%) and a failure to test for HIV during antenatal care (4\%). Only $10 \%$ of factors related to the health system and the main factor was a lack of an effective system to coordinate care between the hospital and the primary care facilities (9\%).

The overall consensus was that $80 \%$ of the HIV infections could have been prevented by implementing the existing Namibian PMTCT recommendation; 5\% of infections happened despite following the recommendations and in $15 \%$ of children it was difficult to decide whether the infection could have been avoided.

\section{Discussion}

The overall transmission rate was very low and suggests that the PMTCT programme in Onandjokwe District was working well. Amongst the $2 \%$ of pregnancies where HIV-positive mothers transmitted the virus the majority of modifiable factors were allocated to the mother in terms of her adherence to use of antiretroviral medication, antenatal care visits, mixed feeding and unsafe sex. A number of modifiable factors were, however, identified in the health services and system. These included a failure to check on or respond to poor adherence in the mother, delayed initiation of ART, failure to test for HIV and poor coordination of care between the hospital and primary care facilities. The proportion of infections that were judged to be avoidable were much higher than in South Africa ( $80 \%$ vs.
$34 \%),{ }^{14}$ although the South African study did not look at adherence to treatment or duration of ART.

In South Africa the majority of modifiable factors were also related to the mothers and similarly $50 \%$ of mothers were not aware of their HIV status at the time they fell pregnant. ${ }^{14}$ Interestingly, twice as many female compared with male infants were infected in Namibia and there is no clear explanation for this. South Africa reported equal numbers of male and female infected infants. $^{14}$

Defaulting and poor adherence amongst mothers was a key finding and was also reported in Malawi where $20 \%$ of patients who initiated ART missed appointments and only $40 \%$ could be traced. ${ }^{23}$ Overall $50 \%$ of the infants in Onandjokwe never received nevirapine, which is slightly more than the $43 \%$ reported in South Africa. ${ }^{15}$ This number can be partly explained by the fact that $25 \%$ of the mothers were only diagnosed postnatally when their children were admitted for sickness and in $6 \%$ nevirapine was out of stock. The results can be compared to South Africa where $26 \%$ of mothers were also tested late for HIV. $^{15}$

Mixed feeding was common (44\%) and appeared much higher than reports from South Africa (6\%). ${ }^{15}$ Again differences might be due to methodological issues; however, such high rates may require further study and intervention.

A third of mothers attended ANC late or not at all, which could make it difficult for mothers to test for HIV and receive a timely PMTCT intervention. This rate, however, is lower than South Africa, where approximately half of the mothers had their first ANC visit in the third trimester. ${ }^{15}$ In the USA $56 \%$ did not receive antenatal care. ${ }^{15,24}$

In addition 19\% of mothers delivered either at home or on transit to the district hospital and might have delayed or missed the PMTCT interventions. In Malawi only $77 \%$ of women who delivered at home could be traced to get PMTCT interventions and none of their children were taken back to health facilities for nevirapine prophylaxis. ${ }^{20}$

Health professionals were responsible for a failure to act on results showing a high HIV viral load and lack of initiative from healthcare workers to trace defaulters. Higher rates of poor viral load monitoring have been reported in South Africa (75\%) and the USA (37\%) as well as failure to take action when a high viral load is observed. ${ }^{15,22} \mathrm{~A}$ failure to respond to virological failure may also expose the infant to resistant HIV strains and negate the value of nevirapine prophylaxis. Missed opportunities to provide ART was seen in 14\% of children, almost similar to a rate of $15 \%$ in South Africa. ${ }^{14}$ Several pregnant mothers had a delay in the initiation of ART (8\%), despite clear Namibian ART guideline recommendations, ${ }^{25}$ although higher rates have also been reported in South Africa, Ghana and Kenya., ${ }^{6,9,15}$ Late maternal HIV testing puts the infant at higher risk of HIV transmission and this issue has also been observed in South Africa (32\% of mothers) and the USA ( $26 \%$ of mothers). ${ }^{14,15,20,24}$

Healthcare system gaps included a lack of coordination of care between district hospitals and primary care facilities, such that the mothers who were expected to come for follow-up could not be identified easily and were not traced if they failed to attend. 


\section{Limitations of the study}

Not all medical records were available and additional factors might have been identified or ranked differently if all records were included in the study. Some of the included records lacked sufficient information to judge whether modifiable factors were present despite the fact that the review team also waited for the mother-child's follow-up visit to elicit information from the patient's and child's road-to-health card. The results of this study depend on the analysis and decision-making of the review team. In cases where there was no consensus, for example if the factor was primarily related to healthcare workers or to patients, the decision was made on the opinion of the majority of the panel. The study involved infants who were already diagnosed with HIV and, therefore, HIV-positive infants who were not yet diagnosed would be excluded from the study.

\section{Recommendations}

The transmission of HIV to an infant should be seen as a critical incident and a review of the records to identify modifiable factors should become institutionalised and not just part of a research study. This will enable ongoing learning and correction of the PMTCT programme's challenges and hopefully realisation of the goal of zero new HIV infections by 2030.

Patient empowerment and engagement is crucial and health education on ART should be provided at different service delivery points such as ANC, ART support counselling teams and through the media to reduce mothers defaulting on their ART. Comprehensive education on the PMTCT approach should include the importance of infant nevirapine prophylaxis, early infant diagnostic tests, feeding options, adherence to medication, and use of condoms during the breastfeeding period. Namibia has an extensive network of health extension workers in the community, and health facilities should work in collaboration with this cadre to provide community education and sensitisation on the importance of attending ANC and if possible to plan to stay near to the health facility when they are due to deliver.

Mothers unable to access healthcare facilities due to long distances should be assisted to create local support groups with the help of health extension workers, whereby they can receive $A R T$ in the community.

Not all primary care providers have attended training in HIV testing and in some facilities patients may be referred to make a diagnosis. Provider initiated testing and counselling (PITC) training should be made available and clinical governance should ensure that all providers are capable of providing this service.

Services for ANC, postnatal care and HIV are still separated within health facilities, which may also contribute to fragmentation of care. Integration of these services should improve access to care, monitoring of viral load in the mothers and early infant diagnosis.

To avoid children missing nevirapine the mother's contacts should be obtained immediately after delivery and a reminder given by phone or health extension worker prior to their follow-up date. Better communication and coordination of care is needed between hospital and primary care facilities to enable this. Each facility should have a standard operating procedure with clear steps on how to offer PMTCT services. Nevirapine should be available at all times; in case of no supply from central medical stores hospital arrangements should be made for buy-out from private pharmacies.

\section{Conclusion}

Although the overall HIV transmission rate from mothers to infants was low, modifiable factors could have prevented transmission in $80 \%$ of children that were infected. Overall $61 \%$ of modifiable factors were related to mothers, $30 \%$ to health professionals and $10 \%$ to the healthcare system. The key modifiable factors related to the mother were poor adherence to ART in themselves or their infant, poor attendance at antenatal care, mixed feeding and unsafe sex during pregnancy. The key modifiable factors related to the health services and system were a failure to check on or respond to poor adherence in the mother, delayed initiation of ART, failure to test for HIV and poor coordination of care between the hospital and primary care facilities. The Onandjokwe district should plan interventions to target these modifiable factors, which may also be relevant to other health districts in Namibia.

Acknowledgements - Thanks are offered to the District HIV Nurse Mentor and Regional Doctor HIV clinical mentor for availing their time and agreeing to be part of review committee to discuss each case and reach consensus.

Disclosure statement - No potential conflict of interest was reported by the authors. The researcher has no conflict of interest in this study; her enthusiasm towards accomplishing this work comes from interest in fighting the HIV pandemic in Namibia and the whole world.

Funding - No funding was received for this study.

Ethical considerations - The research was conducted according to the ethical principles in the Helsinki Declaration, approved by the Health Research Ethics Committee of Stellenbosch University (S16/06/109) and was granted permission by Onandjokwe's district research committee.

\section{ORCID}

Bob Mash (D) http://orcid.org/0000-0001-7373-0774

\section{References}

1. Global plan towards the elimination of new HIV infections among children by 2015 and keeping their mothers alive. [cited 2016 Feb 26]. Available from: http://www.readbag.com/unaids-en-mediaunaids-contentassets-documents-unaidspublication-2011-20110609 -jc2137-global-plan-elimination-hiv-children-en

2. Proposal for Sustainable Development Goals. Sustainable development knowledge platform [cited 2016 Feb 23]. Available from: https://sustainabledevelopment.un.org/focussdgs.html

3. Prevention of mother-to-child transmission (PMTCT) of HIV. [cited 2016 Apr 15]. Available from: http://www.avert.org/professionals/ hiv-programming/prevention/prevention-mother-child

4. World Health Organization. WHO validates elimination of mother-tochild transmission of HIV and syphilis in Cuba [cited 2016 Feb 26]. Available from: http://www.who.int/mediacentre/news/releases/ 2015/mtct-hiv-cuba/en/

5. Caffe S, Perez F, Kamb ML, et al. Cuba validated as the first country to eliminate mother-to-child transmission of human immunodeficiency virus and congenital syphilis: lessons learned from the implementation of the global validation methodology. Sex Transm Dis. 2016;43(12):733-736. doi:10.1097/OLQ.0000000000000528

6. VanDeusen A, Paintsil E, Agyarko-Poku T, et al. Cost effectiveness of option B plus for prevention of mother-to-child transmission of HIV in resource-limited countries: evidence from Kumasi, Ghana. BMC Infect Dis. 2015;15:130. doi:10.1186/s12879-015-0859-2 
7. Bhardwaj S, Carter B, Aarons GA, et al. Implementation research for the prevention of mother-to-child HIV transmission in Sub-Saharan Africa: existing evidence, current gaps, and new opportunities. Curr HIV/AIDS Rep. 2015;12(2):246-255. doi:10.1007/s11904-015-0260-1

8. Shetty AK. Epidemiology of HIV infection in women and children: a global perspective. Curr HIV Res. 2013;11(2):81-92. doi:10.2174/ 1570162X11311020002

9. Thomas TK, Masaba R, Borkowf CB, et al. Triple-antiretroviral prophylaxis to prevent mother-to-child HIV transmission through breastfeeding - the Kisumu breastfeeding study, Kenya: a clinical trial. PLoS Med. 2011;8(3):e1001015. doi:10.1371/journal.pmed.1001015

10. World Health Organization. Consolidated guidelines on the use of antiretroviral drugs for treating and preventing HIV infection [cited 2016 Feb 29]. Available from: http://www.ncbi.nlm.nih.gov/books/ NBK195400/

11. World Health Organization. Guideline on when to start antiretroviral therapy and on pre-exposure prophylaxis for HIV [cited 2016 Feb 26]. Available from: http://apps.who.int/iris/bitstream/10665/186275/1/ 9789241509565_eng.pdf

12. AIDS Info. Recommendations for use of antiretroviral drugs in pregnant HIV-1 infected women for maternal health and interventions to reduce perinatal HIV transmission in the United States [cited 2017 Jun 24]. Available from: https://aidsinfo.nih.gov/guidelines/ html/3/perinatal-guidelines/187/infant-antiretroviral-prophylaxis

13. Republic of Namibia. National guidelines for antiretroviral therapy. 5th ed. [cited 2017 Jun 28], Ministry of Health and Social Service, Namibia. Available from: http://www.methealth.com.na/PDF/2017/ MaxFINAL_Namibia_ART_Guidelines2017.pdf.

14. Woldesenbet $S$, Jackson D, Lombard C, et al. Missed opportunities along the prevention of mother-to-child transmission services cascade in South Africa: uptake, determinants, and attributable risk (the SAPMTCTE). PLoS One. 2015;10(7):e0132425. doi:10.1371/ journal.pone.0132425

15. Kendall C, Claessens L, Dorward J, et al. Reasons for failure of prevention of mother-to-child HIV transmission in a rural South African district hospital. South Afr J HIV Med. 2015;16(1):a365. doi:10.4102/ sajhivmed.v16i1.365

16. UNAIDS. Progress report on the global plan towards the elimination of new HIV infections among children and keeping their mothers alive [cited 2018 Jun 27]. Available from: http://www. unaids.org/en/resources/documents/2015/JC2774_2015ProgressRep ort_GlobalPlan

17. Buchanan AM, Cunningham CK. Advances and failures in preventing perinatal human immunodeficiency virus infection. Clin Microbiol Rev. 2009;22(3):493-507. doi:10.1128/CMR.00054-08

18. Audureau $\mathrm{E}$, Kahn JG, Besson $\mathrm{M}-\mathrm{H}$, et al. Scaling up prevention of mother-to-child HIV transmission programs in sub-Saharan African countries: a multilevel assessment of site-, program- and countrylevel determinants of performance. BMC Public Health. 2013;13 (1):286. doi:10.1186/1471-2458-13-286

19. Paintsil E, Andiman WA. Update on successes and challenges regarding mother-to-child transmission of HIV. Curr Opin Pediatr. 2009;21 (1):94-101. doi:10.1097/MOP.0b013e32831ec353

20. Mdala JF, Mash R. Causes of mortality and associated modifiable health care factors for children ( $<5$-years) admitted at onandjokwe hospital, Namibia. African J Prim Heal Care Fam Med. 2015;7(1):1-8. doi:10.4102/phcfm.v7i1.840

21. Sialubanje $\mathrm{C}$, Massar $\mathrm{K}$, Hamer $\mathrm{DH}$, et al. Reasons for home delivery and use of traditional birth attendants in rural Zambia: a qualitative study. BMC Pregnancy Childbirth. 2015;15:123. doi:10.1186/s12884015-0652-7.

22. Namibian Ministry of Health and Social Services Directorate of Special Programmes. National guidelines for antiretroviral therapy 2010 [cited 2017 Oct 15]. Available from: http://www.who.int/hiv/ pub/guidelines/namibia_art.pdf

23. Tweya H, Gugsa S, Hosseinipour M, et al. Understanding factors, outcomes and reasons for loss to follow-up among women in option $\mathrm{B}+$ PMTCT programme in Lilongwe, Malawi. Trop Med Int Heal 2014;19 (11):1360-1366. doi:10.1111/tmi.12369

24. Camacho-Gonzalez AF, Kingbo M-H, Boylan A, et al. Missed opportunities for prevention of mother-to-child transmission in the United States. AIDS. 2015;29(12):1511-1515. doi:10.1097/QAD. 0000000000000710

25. Namibia national ART guidelines. 2014 [cited 2016 Mar 1]. Available from: http://preventcrypto.org/wp-content/uploads/2012/07/Namib ia-National-ART-guidelines-2014.pdf

Received: 11-07-2018 Accepted: 24-08-2018 